\title{
Parameterizing ice cloud inhomogeneity and the overlap of inhomogeneities using cloud radar data
}

\author{
Robin J. HogAN ${ }^{1}$ AND ANTHONY J. ILLINGWORTH \\ Department of Meteorology, University of Reading, United Kingdom
}

September 13, 2002

\footnotetext{
${ }^{1}$ Corresponding author address: Department of Meteorology, Earley Gate, PO Box 243, Reading RG6 6BB, United Kingdom.

E-mail: r.j.hogan@reading.ac.uk.
} 


\begin{abstract}
Cloud variability on scales smaller than the gridbox size of numerical forecast and climate models is believed to be important in determining the radiative effects of clouds, and increasingly attempts are being made to parameterize these fluctuations in the radiation schemes of current models. In order to calculate the radiative effects of an inhomogeneous cloud a model needs to know not only the degree of variability within a gridbox, but also the degree to which the inhomogeneities in vertically adjacent levels are overlapped. In this paper these two parameters are derived for ice clouds from an 18-month mid-latitude 94-GHz cloud radar dataset and parameterized in terms of horizontal gridbox size $(d)$, the vertical shear of the horizontal wind $(s)$ and the vertical position in the cloud. The vertical decorrelation length $\Delta z_{0}$ (i.e. the depth over which the correlation coefficient of either ice water content or optical extinction coefficient in separate vertical levels falls to $e^{-1}$ ) is found to be well represented in the mean by

$$
\log _{10} \Delta z_{0}=0.3 \log _{10} d-0.031 s-0.315
$$
\end{abstract}

where $\Delta z_{0}$ and $d$ are in $\mathrm{km}$ and $s$ is in $\mathrm{m} \mathrm{s}^{-1} \mathrm{~km}^{-1}$. As expected, higher shear results in more rapid decorrelation, although the rms deviation from this expression is around a factor of 2.5. It is found that the probability distribution of ice water content within a gridbox is usually well represented by a lognormal or gamma distribution. The fractional variance in ice water content $\left(f_{\text {IWC }}\right)$ may be expressed to within a factor of two by

$$
\log _{10} f_{\mathrm{IWC}}=0.3 \log _{10} d-0.04 s-0.93
$$

valid for $d<60 \mathrm{~km}$, above which $f_{\text {IWC }}$ is constant with increasing $d$. The expression for the fractional variance of visible extinction coefficient is the same except with the -0.93 term replaced by -0.96 . The $s$ dependence indicates a tendency for increased shear to result in decreased cloud variability. This can be explained by the presence of ice fallstreaks in a sheared flow: a parcel of air in the middle of a cloud is alternately fed from above by ice-rich and ice-poor air, resulting in a homogenization of the layer at a rate dependent on the shear. A more complicated formula is derived to express the dependence of $f_{\text {IWC }}$ on the vertical position within the cloud; it is found that 
fractional variance tends to be largest at cloud top and decrease into the interior before increasing again in the lowest third of the cloud. Thicker clouds tend to have lower fractional variance. No significant dependence on temperature or absolute altitude was found for either $f_{\text {IWC }}$ or $\Delta z_{0}$. 


\section{Introduction}

The importance of ice clouds on the earth's radiation budget is well recognized (Liou 1986), and increasingly attention is focusing on the role of small-scale cloud variability and how to represent it in models (e.g. Liou and Rao 1996; Donner et al. 1997). Most current forecast and climate models represent the fractional cloudiness of a model gridbox either diagnostically from the relative humidity (e.g. Slingo 1987; Smith 1990; Lohmann and Roeckner 1996), or as a prognostic variable (Tiedtke 1993). Recently, Tompkins (2002) proposed a more advanced scheme which also carries the variance and skewness of the total water content (vapor and condensed phases) as prognostic variables; cloud fraction and condensed water content are then determined by splitting the probability distribution function (PDF) of total water into cloudy and cloud-free components based on the saturation specific humidity. However, the implied distribution of condensed water content in the cloudy part of the box is not currently used in the other parameterizations of these models, which usually treat clouds as horizontally homogeneous.

For boundary-layer clouds, the primary effect of cloud inhomogeneity is to reduce the albedo relative to that computed using the plane-parallel approximation. In an attempt to correct this problem in the model of the European Centre for Medium Range Weather Forecasts, Tiedtke (1996) used the findings of Cahalan et al. (1994) and simply multiplied water content by 0.7 in the calculation of albedo. Barker et al. (1996) proposed a more sophisticated method which involves representing the distribution of optical depth by a gamma distribution function, and validated it using high-resolution satellite imagery. However, if models do not include the effects of cloud variability in all their parameterizations then significant microphysical and thermodynamical biases can also occur (Larson et al. 2001).

This paper addresses the issue of sub-gridscale inhomogeneity in ice clouds, which has generally received much less attention than liquid cloud inhomogeneity. Ice clouds are generally quite deep, occupying several vertical gridboxes, so a single path-integrated satellite estimate of cloud structure is unsatisfactory. Instead, we appeal to the new technology of cloud radar which provides range-resolved data of cloud structure from the ground and, unlike instruments that operate at op- 
tical wavelengths, suffers virtually no attenuation in ice clouds. In the last few years the ability of these instruments to detect cloud boundaries has been exploited to evaluate model representation of cloud occurrence (Mace et al. 1998), cloud fraction (Hogan et al. 2001) and cloud overlap (Hogan and Illingworth 2000). The ability of radar to estimate cloud variables such as ice water content (IWC) was used by Pomroy and Illingworth (2000) and Fu et al. (2000) to address the specific problem of the emissivity bias in models due to ice cloud variability.

Our intention here is to use $94-\mathrm{GHz}$ cloud radar to provide the essential data on ice cloud variability that is needed by the newer model parameterizations that attempt to represent subgridscale cloud structure. This information could be used either as the basis for a diagnostic parameterization for the variance of ice water content, or to evaluate schemes that carry variance as a prognostic variable, such as that of Tompkins (2002). Brown et al. (1995) estimated that IWC could be derived from radar reflectivity factor $(Z)$ with an error of around a factor of two (the typical range of IWC we wish to measure being around three orders of magnitude). Liquid water clouds, on the other hand, often contain low concentrations of 'drizzle' droplets which can dominate $Z$ while containing negligible liquid water, with the result that $Z$ is often essentially unrelated to stratocumulus liquid water content (Fox and Illingworth 1997). We therefore restrict our analysis to ice clouds.

The first parameter to be estimated is the fractional variance of ice water content within a model gridbox, which we define as

$$
f_{\mathrm{IWC}}=\left(\sigma_{\mathrm{IWC}} / \overline{\mathrm{IWC}}\right)^{2}
$$

where $\sigma$ denotes standard deviation. Similarly, the fractional variance of visible extinction coefficient $\alpha$ is defined as

$$
f_{\alpha}=\left(\sigma_{\alpha} / \bar{\alpha}\right)^{2}
$$

The relationship between fractional variance and the parameters used to describe gamma and lognormal distributions is outlined in section 2. Then in section 3, aircraft data taken during the European Cloud Radiation Experiment (EUCREX) are used to derive the appropriate functions relating radar reflectivity to IWC and $\alpha$. 
The other important parameter, in addition to fractional variance, is the vertical decorrelation length, $\Delta z_{0}$, which is the depth over which the correlation coefficient of IWC in separate vertical layers falls to $e^{-1}$. This is important because rapid decorrelation makes a cloud much more effective at blocking outgoing thermal radiation and reflecting incoming visible radiation, compared with the case of the inhomogeneities being stacked above each other, even though the total water content may be the same. Hogan and Illingworth (2000) used radar data to derive a similar parameter for describing 'cloud overlap' (Morcrette and Fouquart 1986; Liang and Wang 1997) for use in current models, and it should be stressed that the two are not necessarily the same; the decorrelation length of Hogan and Illingworth (2000) was derived considering cloud boundaries alone, while here we are considering the overlap characteristics of the in-cloud fluctuations of water content. Nonetheless, the two extremes of $\Delta z_{0}=0$ and $\Delta z_{0}=\infty$ can be thought of as being analogous to the 'random' and 'maximum' overlap assumptions made in models, respectively.

In section 4 we present radar observations of a thick ice cloud and demonstrate the effects of gridbox size and wind shear on the parameters $f_{\text {IWC }}$ and $\Delta z_{0}$. The suitability of gamma and lognormal distributions for fitting the observed PDFs is investigated. Then in section 5, 18 months of near-continuous data taken by the same instrument are analyzed to obtain empirical relationships for these parameters.

\section{Analytical probability distribution functions}

Here we show how fractional variance is related to the parameters describing analytical PDFs used to represent the distribution of IWC and $\alpha$ within a gridbox. Barker et al. (1996) used a gamma distribution to represent the optical depth $(\tau)$ distribution of boundary-layer stratocumulus and cumulus as inferred from satellite:

$$
p(\tau)=\frac{1}{\Gamma(\nu)}\left(\frac{\nu}{\bar{\tau}}\right)^{\nu} \tau^{\nu-1} \exp \left(-\frac{\nu \tau}{\bar{\tau}}\right)
$$

where $v=\left(\bar{\tau} / \sigma_{\tau}\right)^{2}$ and $\Gamma(v)$ denotes the gamma function. If the same distribution is used for IWC instead of $\tau$, then we have $v=f_{\mathrm{IWC}^{-1}}$. 
A lognormal distribution for IWC has the form

$$
p(\mathrm{IWC})=\frac{1}{(2 \pi)^{\frac{1}{2}} \sigma_{0} \mathrm{IWC}} \exp \left[-\frac{\ln \left(\mathrm{IWC} / \mathrm{IWC}_{0}\right)^{2}}{2 \sigma_{0}^{2}}\right] .
$$

The parameters of the distribution, $\mathrm{IWC}_{0}$ and $\sigma_{0}$, may be defined in terms of $\overline{\mathrm{IWC}}$ and $f_{\mathrm{IWC}}$ as follows

$$
\begin{aligned}
\mathrm{IWC}_{0} & =\overline{\mathrm{IWC}}\left(f_{\mathrm{IWC}}+1\right)^{-\frac{1}{2}} ; \\
\sigma_{0}^{2} & =\ln \left(f_{\mathrm{IWC}}+1\right) .
\end{aligned}
$$

Thus the results in this paper may be easily compared to other papers that express cloud variability in a different way. The suitability of gamma and lognormal distributions for parameterizing the IWC distribution of real clouds is studied in section 4c.

\section{Deriving relationships from the EUCREX dataset}

\section{a. Accounting for the small ice particles}

To determine the appropriate expressions relating radar reflectivity to IWC and $\alpha$ we follow Brown et al. (1995) and use size spectra recorded by the UK Met Office C-130 aircraft in midlatitude ice clouds during EUCREX. The dataset consists of over 10000 5-s averaged size spectra measured by the $2 \mathrm{D}$ cloud and precipitation probes, which cover the diameter range 25 to $6400 \mu \mathrm{m}$. However, the 2D cloud probe (2D-C) is known to be somewhat unreliable for measuring particles smaller than $100 \mu \mathrm{m}$ (Heymsfield and Baumgardner 1985). Francis et al. (1998) used replicator measurements of the small crystals taken during a few runs of EUCREX to suggest a correction for the whole dataset, which involves fitting a gamma distribution to the lower end of the measured size distribution. The gamma distribution was constrained to have a modal diameter of $6 \mu \mathrm{m}$, to have the same concentration of particles of diameter $100 \mu \mathrm{m}$ as measured by the 2D-C, but to have five times more particles of diameter $25 \mu \mathrm{m}$. Note that diameter here refers to the diameter of a circle with the same cross-sectional area as the particle image measured by the probe. The intention of Francis et al. was to produce a 'pessimistic' estimate of the under-counting of the small particles, and in the example plotted in their paper the replicator size spectrum for a 
diameter of $25 \mu \mathrm{m}$ was only 3-3.5 times higher than the concentration measured in the first bin of the $2 \mathrm{D}-\mathrm{C}$. The one or two other runs for which replicator data were available showed a much smaller difference between the two spectra (P. N. Francis, personal communication). McFarquhar and Heymsfield (1997) compared the ice water content contained in the particles with a maximum dimension smaller than $90 \mu \mathrm{m}$ (hereafter $\mathrm{IWC}_{<90}$ ) as measured by the the $2 \mathrm{D}-\mathrm{C}$ and the Video Ice Particle Sampler (VIPS). From Fig. 20a of their paper we estimate that the 2D-C probe underestimated IWC $<90$ by a factor of 2.5 , on average, although there was considerable scatter in their data and on some occasions the 2D-C measured higher values than VIPS.

With these considerations in mind, we fit a gamma distribution to the small particles in the same manner as Francis et al. (1998), but constrain the fitted distribution to be only a factor of two higher than the measured distribution for $25-\mu \mathrm{m}$ particles. This increases $\mathrm{IWC}_{<90}$ by around a factor of 2.5, in agreement with the findings of McFarquhar and Heymsfield (1997).

From these modified distributions we calculate IWC using the mass-area relationship of Francis et al. (1998), while $\alpha$ is defined to be simply twice the total cross-sectional of particles per unit volume. Radar reflectivity at $94 \mathrm{GHz}$ is calculated in the same manner as Brown et al. (1995) and Hogan and Illingworth (1999); the crystals are approximated as homogeneous ice-air spheres with a diameter $D_{\mathrm{m}}$ equal to the mean of the maximum particle dimensions measured parallel and perpendicular to the probe photodiode array, their density is determined from the mass- $D_{\mathrm{m}}$ relationship of Brown and Francis (1995), and Mie theory is applied. It should be noted that $D_{\mathrm{m}}$ is systematically larger than the 'equivalent area' diameter, but is believed to better represent the extremities of the particle which are important when the scattering at $94 \mathrm{GHz}$ departs from the Rayleigh approximation.

\section{b. The SD line}

We wish to derive relationships of the form

$$
X=c Z^{b}
$$


where $X$ is either IWC or $\alpha$, and $Z$ is in $\mathrm{mm}^{6} \mathrm{~m}^{-3}$. Since in this paper we are considering the fractional variance of IWC and $\alpha$, only the exponent $b$ in (7) is important. The $\Delta z_{0}$ parameter is also completely insensitive to the $c$ coefficient. Therefore the results are unaffected by any error in radar calibration.

Numerous Z-IWC power law relationships exist in the literature, and are typically derived by performing a linear regression between the two parameters in logarithmic space. The slope of the regression line is then equal to $b$. This approach produces the best estimate of IWC for any given measurement of $Z$, but in this study we are really interested in obtaining the best estimate of the fractional variance of IWC given a measurement of the fractional variance of $Z$. Since fractional variance is equivalent to the variance of the logarithm, we require our best-fit line to have a slope $b=\sigma_{\log \text { IWC }} / \sigma_{\log Z}$, where $\sigma$ denotes standard deviation. However, according to statistical theory, the slope of the regression line is actually $r \sigma_{\log \text { IWC }} / \sigma_{\log Z}$, where $r$ is the correlation coefficient between $\log$ IWC and $\log Z$. This simply expresses the definition of the correlation coefficient, that with each one-standard-deviation increase in one of the variables, there is an increase of only $r$ standard deviations in the other, on the average. Invariably $r$ is significantly less than unity, so relationships derived by linear regression in logarithmic space will tend to result in an underestimate of $f_{\mathrm{IWC}}$ by a factor of $r^{2}$. We therefore use the $S D$ line $e^{1}$ rather than the regression line as the basis for relationships between $Z$ and other parameters.

A further consideration is that fits to an entire dataset include the variance associated with changing altitude and temperature, whereas each retrieved value of $f_{\text {IWC }}$ in this study will correspond to one particular altitude and temperature. The SD line is therefore calculated separately for each horizontal aircraft run in the EUCREX dataset.

The thin solid lines in Fig. 1a depict the SD lines for each of the 115 aircraft runs of EUCREX (two of the runs consisted of fewer than ten 5-s samples and were rejected). The 'best' SD line, shown by the thick solid line, passes through the point of means, i.e. $(\overline{\log I W C}, \overline{\log Z})$, and has a

\footnotetext{
${ }^{1}$ In statistics, the 'standard deviation line', or SD line, for abscissa $x$ and ordinate $y$ is defined to have a slope of $\sigma_{y} / \sigma_{x}$ and pass through the point $(\bar{x}, \bar{y})$.
} 
slope equal to the mean of the slopes of the individual runs. Its formula is

$$
\mathrm{IWC}=0.241 Z^{0.868} \mathrm{~g} \mathrm{~m}^{-3}
$$

where $Z$ has the units $\mathrm{mm}^{6} \mathrm{~m}^{-3}$. The range of exponents in the individual runs is indicated in Fig. 1b. The standard deviation of $0.18( \pm 21 \%)$ suggests a random rms error in $f_{\text {IWC }}$ retrieved using $94-\mathrm{GHz}$ radar of $\pm 42 \%$. The regression line (shown by the thick dashed line in Fig. 1a) has a significantly smaller slope of 0.693 , and is much closer to values previously reported in the literature (see Liu and Illingworth 2000). The effect of not performing the small-particle correction described in section $3 \mathrm{a}$ is to increase the slope of the regression line by $10 \%$, although the slope of the best SD line in (8) is changed by only $2 \%$.

It should be stressed that (8) is not suitable for retrieving a best estimate of the absolute values of IWC, nor should it be used at frequencies other than $94 \mathrm{GHz}$. It may be used in the retrieval of $\Delta z_{0}$, however, since $\Delta z_{0}$ is very insensitive to the exact form of the $Z$-IWC relationship, or indeed to which variable is being correlated.

The analogous relationship for visible extinction coefficient is derived in exactly the same way (see Fig. 2):

$$
\alpha=0.00691 Z^{0.841} \mathrm{~m}^{-1}
$$

The error in retrieved $f_{\alpha}$ is around $\pm 45 \%$. Due to the lower correlation between $Z$ and $\alpha$, the slope of the regression line is significantly lower at 0.558 . It may appear counter-intuitive that the exponents in (8) and (9) are so similar when IWC and $\alpha$ represent different moments of the size distribution, but this is due to several of factors. Firstly, IWC should not be regarded as the third moment of the size distribution, but a moment between the second and third (and therefore closer to $\alpha$ ), because of the tendency for ice particle density to decrease with size (Brown and Francis 1995; Francis et al. 1998). Secondly, much of the variability in the various moments of the distribution is not due to changes in mean particle size, but to changes in total number concentration which affect each moment equally. Moreover, by calculating the SD lines separately for each horizontal aircraft run, the contribution of particle size variability is minimized since size varies predominantly in the vertical. 
It is straightforward to deduce how the exponent will affect our retrieval of $f_{\text {IWC }}$ : (8) implies that the fractional standard deviation of IWC within a gridbox will be close to 0.868 times that of $Z$ itself. Therefore, to change to a new exponent in (8) simply involves scaling any derived formulae for $f_{\text {IWC }}$ by the square of the ratio of the new and the old exponents. Likewise, the fractional variance of other bulk cloud parameters that can be related to $Z$ by a power law may be derived simply from $f_{\mathrm{IWC}}$. Hence, from the ratio of exponents in (9) and (8) it can be seen that $f_{\alpha}=0.939 f_{\text {IWC }}$.

It should be mentioned that there is a slight dependence of the slope of the SD lines on temperature; the correlation between the slope $b$ of the Z-IWC relationship and temperature is 0.40 , while for the $Z$ - $\alpha$ relationship it is 0.18 . The reason for the correlation is that at higher temperatures the ice particles tend to be larger. In the Rayleigh scattering regime this would be sufficient only to change the $c$ coefficient in (7), but at high frequencies such as $94 \mathrm{GHz}$, the largest particles scatter outside the Rayleigh regime which changes the slope as well. Because of the inherent uncertainties in our representation of the non-Rayleigh effects, and the low correlation between $b$ and temperature, we choose not to include this effect in our relationships.

\section{27 December 1999 case study}

\section{a. Fallstreak structure}

Figure 3a shows a 4-hr time-height section of $Z$ measured in thick ice cloud by the verticallypointing 94-GHz Galileo radar, located at Chilbolton, England $\left(51.145^{\circ} \mathrm{N}, 1.437^{\circ} \mathrm{W}\right)$. The data were taken on 27 December 1999 and the resolution is $30 \mathrm{~s}$ and $60 \mathrm{~m}$. A very distinct fallstreak structure is evident throughout the depth of the cloud. Above around $7 \mathrm{~km}$ the cloud inhomogeneities associated with the fallstreaks appear to be close to vertically aligned, while below this height they become much more slanted. This structure can be understood on examination of the 11 UTC wind profile in Fig. 3c (obtained from the mesoscale version of the UK Met Office Unified Model), which shows a strong zonal jet exceeding $60 \mathrm{~m} \mathrm{~s}^{-1}$ between 7 and $8 \mathrm{~km}$ altitude where the shear was less than $5 \mathrm{~m} \mathrm{~s}^{-1} \mathrm{~km}^{-1}$. Immediately below $6.9 \mathrm{~km}$, however, the shear exceeds $20 \mathrm{~m} \mathrm{~s}^{-1} \mathrm{~km}^{-1}$. At an altitude of 5-6 km, the gradient of the fallstreaks in the time-height image 
is between 10 and 13 minutes per $\mathrm{km}$; from the model winds this implies that the fallstreaks had a horizontal-vertical aspect ratio of 25-30. The wind vector difference of around $30 \mathrm{~m} \mathrm{~s}^{-1}$ between the jet and an altitude of $5.5 \mathrm{~km}$ is therefore consistent with an ice fall speed of around $1 \mathrm{~m} \mathrm{~s}^{-1}$.

This simple comparison between model and observations demonstrates qualitatively the importance of shear in determining the orientation of fallstreaks, and hence the correlation of horizontal cloud inhomogeneities from one vertical level to the next.

\section{b. Fractional variance of IWC}

We next consider how the inhomogeneity evident in this cloud would appear in a model. Reflectivity factor is first averaged to $0.3 \mathrm{~km}$ in the vertical, imitating the highest resolutions that are expected to be achieved by operational mesoscale models in the next few years. Ice water content is then calculated using (8) and is shown in a linear scale in Fig. 3b. We consider a horizontal gridbox size of $50 \mathrm{~km}$ by using the model wind speeds to select periods of time between 10 UTC and 14 UTC equivalent to this distance. Only fully cloudy boxes are considered, because a model implementing a parameterization for cloud variability would doubtless have also implemented an ordinary cloud fraction scheme to cope with partially cloudy gridboxes. It should be noted that the direct correspondence between time and space assumed here (and throughout the paper) may 'bias in' variations due to cloud temporal evolution, especially in low wind speed conditions or for larger gridbox sizes. However, many models only call their radiation schemes every three hours, in which time considerable evolution can occur. It is therefore possible that too little cloud evolution is being included in our results, rather than too much.

A value for $f_{\mathrm{IWC}}$ is calculated for each $50 \times 0.3 \mathrm{~km}$ box, and Fig. $3 \mathrm{~d}$ shows the mean and standard deviation at each height. Because of the increase in wind speed with height, the data that make up the 50-km samples are around three times better resolved at the bottom of the cloud than the top. This should not affect the results since the variability occurs predominately at scales much larger than the 30 -s resolution of the data. It can be seen that mean $f_{\text {IWC }}$ decreases from 0.8 near cloud top to 0.1 at $5.7 \mathrm{~km}$. Looking at Fig. 3a, it seems that after formation at cloud top the fallstreaks remained relatively coherent in the first $1-2 \mathrm{~km}$ where the vertical wind shear was 
low. Then in the region of much higher shear below around $7 \mathrm{~km}$, much of the fine structure was lost, particularly after 12 UTC, with an ensuing fall in $f_{\text {IWC }}$. This can be explained by considering a parcel in the middle of the sheared region; the differential velocity with the layer above would result in it being alternately fed by ice-rich and ice-poor air, resulting in a homogenization of the horizontal ice distribution at a rate dependent on the magnitude of the shear.

Below $5.7 \mathrm{~km}$, the mean $f_{\mathrm{IWC}}$ increases again toward cloud base. This is due to the base of the cloud becoming steadily lower during the four hour period, with the result that significant horizontal gradients are present across the gridboxes near cloud base. Also, strong small-scale variability is present at cloud base after around 1330 UTC. This is most likely to be instability generated by the latent heat release associated with sublimation, and is not uncommon at the base of thick ice clouds.

The distinctive behavior evident in Fig. 3 d suggests that $f_{\text {IWC }}$ could be parameterized effectively in terms of wind shear and distance from cloud top or cloud base. However, the standard deviation of $f_{\text {IWC }}$ in Fig. $3 \mathrm{~d}$ increases from $\pm 25 \%$ near cloud top to $+300 \% /-75 \%$ below $5 \mathrm{~km}$. So, even in a single cloud with similar shear conditions persisting throughout the period that it is observed, different $f_{\text {IWC }}$ values are able to evolve. This limits the ability of a parameterization to predict $f_{\text {IWC }}$ precisely. Indeed, we should conclude that the cloud fractional variance in individual model gridboxes (particularly below cloud top) is impossible to predict accurately based on the information available to large-scale models, but that the mean fractional variance over many realizations may be possible to predict accurately. For the purposes of climate modeling this is probably sufficient, especially given that most current models effectively assume that $f_{\text {IWC }}=0$ in all ice clouds. The same reasoning holds for current 'cloud overlap' schemes; the overlap behavior of individual clouds can vary greatly, but if models can capture the mean overlap characteristics of clouds then the corresponding radiative fluxes should be unbiased.

The range of $f_{\text {IWC }}$ values observed in this case study is comparable to values that may be derived from the results of other researchers. Smith and DelGenio (2001) plotted aircraft measurements of $\sigma_{\mathrm{IWC}}$ against $\overline{\mathrm{IWC}}$ in cirrus for runs between 50 and $300 \mathrm{~km}$ in length. Figure 7a of their paper indicates values of $f_{\mathrm{IWC}}$ ranging between 0.09 and 1.5 , with a median of around 
0.5. Presumably the larger values corresponded to the longer aircraft runs. Barker et al. (1996) examined the distribution of $\tau$ in $60 \times 60-\mathrm{km}$ regions of boundary-layer cloud observed by satellite, and for overcast stratocumulus reported $v$ (defined in Eq. 3) to be $7.98 \pm 6.29$, equivalent to a fractional variance of optical depth, $f_{\tau}$, of $0.13 \pm 0.10$. For broken stratocumulus they found $f_{\tau}=0.83 \pm 0.42$. It is interesting that this range of values for $f_{\tau}$ is similar to the $0.1-0.8$ range for $f_{\mathrm{IWC}}$ found in Fig. 3c, despite the fact that quite different parameters and cloud types are being compared.

\section{c. Probability distribution functions}

In this section the sub-gridscale distribution of ice water content is investigated. Figure 4 shows PDFs of IWC measured in three 50-km boxes at different heights in the cloud, each centered on 1230 UTC. As before the model winds have been used to determine the appropriate sample time. The values of $f_{\mathrm{IWC}}$ are shown in each panel. Overlayed on each measured PDF are lognormal and gamma distribution functions, fitted such that they have the same mean and variance as the original data. In these cases there is little difference between the two functions and both fit the data reasonably well, although there is some bimodality evident at $4.65 \mathrm{~km}$.

Figure 5 shows PDFs at the same heights as in Fig. 4, but using boxes with a horizontal size of $250 \mathrm{~km}$. The occurrence of larger values of $f_{\text {IWC }}$ indicates the need to include gridbox size in any parameterization for cloud variability. Again, the PDFs at 6.45 and $8.25 \mathrm{~km}$ are well represented by both lognormal and gamma distributions, but this time the PDF at 4.65 is very poorly represented by either. A 'top hat' function would probably describe the distribution better. The reason is that most of the variance at this altitude is not due to small-scale fluctuations but to a trend in IWC across the gridbox, from zero (i.e. cloud-free air) at the beginning of the period to $0.7 \mathrm{~g} \mathrm{~m}^{-3}$ in the thick fallstreaks at the end. A model may be able to use the resolved horizontal gradient in IWC to determine how much variance within each box is due to such a trend, but this is only likely to be necessary for relatively low resolution models. 


\section{d. Vertical correlation of ice water content}

The next step is to look at the correlation of the horizontal structure with height. The IWC between 10 UTC and 14 UTC is analyzed separately in the height ranges 6.9 to $8.9 \mathrm{~km}$ (the 'low shear' case) and 4.9 to $6.9 \mathrm{~km}$ (the 'high shear' case). A model horizontal gridbox size of $50 \mathrm{~km}$ is simulated by dividing the data above $6.9 \mathrm{~km}$ (where the mean wind speed was $63 \mathrm{~m} \mathrm{~s}^{-1}$ ) into 13-min boxes and the data below this height (where the mean wind speed was $38 \mathrm{~m} \mathrm{~s}^{-1}$ ) into 22-min boxes.

In each column of boxes, the correlation coefficient, $\rho$, is computed for the IWC of each level with each other level, neglecting partially cloudy boxes, and with no 'double-counting'. The circles in Fig. 6 depict the mean $\rho$ versus vertical separation, $\Delta z$, for the low shear and high shear regions. In both cases the correlation of the horizontal structure decreases rapidly with $\Delta z$, and for $\Delta z>1 \mathrm{~km}$ it is essentially uncorrelated. The difference in results is negligible if the correlation is performed on $\ln ($ IWC) instead of IWC. The solid lines in Fig. 6 are simple inverse-exponential expressions of the form

$$
\bar{\rho}=\exp \left(-\frac{\Delta z}{\Delta z_{0}}\right)
$$

with $\Delta z_{0}$ chosen to give the correct $\bar{\rho}$ for adjacent layers, $0.3 \mathrm{~km}$ apart. The corresponding decorrelation lengths, $\Delta z_{0}$, are $0.68 \mathrm{~km}$ and $0.35 \mathrm{~km}$ for the low and high shear cases respectively. Hence, as expected, high shear produces a much more rapid decorrelation with height.

This analysis is similar to that performed by Hogan and Illingworth (2000) in their determination of an $e$-folding length-scale over which the overlap of clouds in a partially cloudy gridbox becomes random, but there are some interesting differences. Firstly, they reported values in the range $1.40-2.93 \mathrm{~km}$, depending on the resolution of the gridbox, whereas our values are a factor of 4 smaller. This presumably reflects the larger horizontal and vertical scales that cloud boundaries exist on compared with the scales of sub-cloud variability. Secondly, they reported that several days of data were necessary to obtain something approaching an inverse-exponential relationship, while as little as $4 \mathrm{hrs}$ of data seem to be sufficient here.

At this point we should consider whether an inverse-exponential is really the best way of char- 
acterizing the relationship between correlation coefficient and vertical separation. Although the inverse-exponential falls within all the error bars of the observations (indicating the range of $\rho$ that was actually measured), the biggest difference between it and the mean $\rho$ occurs for a vertical separation of $0.6 \mathrm{~km}$, where $\rho$ was significantly less than that indicated by the inverse-exponential. In fact it seems that the first $0.6 \mathrm{~km}$ would be much better characterized by a linear decrease of $\rho$ with $\Delta z$ (indicated by the dashed lines in Fig. 6). This is presumably because the decorrelation is due to fallstreaks, coherent over several kilometers in the vertical, which pass through the columns of boxes at an angle, rather than being due to some genuinely random process. It is interesting that the same phenomenon appears to be present to a small extent in the randomization of cloud overlap (Hogan and Illingworth 2000, Fig. 4). Despite this behavior, the results presented in the remainder of this paper are in terms of decorrelation length because of its appeal in conceptualizing the phenomenon of cloud decorrelation in the vertical, and because it is independent of the vertical resolution under consideration. The reader should be aware, however, that it is calculated simply from the correlation coefficient between layers $0.3 \mathrm{~km}$ apart.

\section{Analysis of 18 months of radar data}

We now extend the analysis performed in the last section to the near-continuous observations taken at Chilbolton by the 94-GHz Galileo cloud radar between May 1999 and October 2000. Again, the derived IWC data are used at a resolution of $0.3 \mathrm{~km}$ in the vertical and $30 \mathrm{~s}$ temporally, and only fully cloudy boxes are considered. The data are analyzed in boxes of duration varying between 5 mins and 3 hrs. Because of the possibility of variable attenuation by rain, which would tend to increase the correlation between the layers above, data taken by a drop-counting rain gauge at Chilbolton were used to reject whole columns of data from the analysis whenever rain was detected. Attenuation by the ice clouds themselves is negligible (Hogan and Illingworth 1999). For each box the fractional variance of ice water content, $f_{\text {IWC }}$, is computed, and for every pair of vertically adjacent boxes we calculate the correlation correlation coefficient, $\rho$.

These values are then combined with data from the Unified Model over Chilbolton, which consist of the six-hourly analyses and the intervening hourly forecasts. The model wind speeds are 
used to convert the 15 different temporal resolutions into horizontal resolutions. Clouds warmer than $-5^{\circ} \mathrm{C}$ are rejected to prevent contamination of the results by the melting layer. The magnitude of the wind shear is extracted at the height of every $f_{\text {IWC }}$ and $\rho$ observation.

We first classify the resulting 221659 data points by horizontal box size, $d$, and wind shear, $s$. Nine $d$ classes are used, the mean sizes of which are spaced logarithmically between 2 and $300 \mathrm{~km}$. The data are then further divided according to $s$ into $5 \mathrm{~m} \mathrm{~s}^{-1} \mathrm{~km}^{-1}$ intervals, and mean $f_{\text {IWC }}$ is calculated in each class. The decorrelation length is calculated from the median $\rho$ in each class.

These values are plotted in log-log space in Fig. 7 as a function of $d$ and $s$. Means calculated from fewer than 100 data points have not been plotted because of doubts over their statistical representativity. These points represent means of very scattered data as indicated by the error bars; the standard deviation of $f_{\mathrm{IWC}}$ is around $+100 \% /-50 \%$, and the standard deviation of $\Delta z_{0}$ is around $+150 \% /-60 \%$. It should be noted that this is greater than the estimated error in the retrieval associated with uncertainties in the $Z$-IWC relationship. Nonetheless, some clear trends are evident. There seem to be approximate power-laws relating both $f_{\text {IWC }}$ and $\Delta z_{0}$ to $d$, while the dependence on $s$ is closer to inverse-exponential. Up to $d=60 \mathrm{~km}$, the $f_{\mathrm{IWC}}$ data are quite well fitted by the following expression, shown by the dashed lines in Fig. 7a:

$$
\log _{10} f_{\mathrm{IWC}}=0.3 \log _{10} d-0.04 s-0.93
$$

where $d$ is in $\mathrm{km}$ and $s$ has the units $\mathrm{m} \mathrm{s}^{-1} \mathrm{~km}^{-1}$. At around $d=60 \mathrm{~km}$, the observations suggest the beginnings of a scale break, beyond which mean $f_{\text {IWC }}$ reaches an upper limit of around 0.4. Over 8000 events are present with $d>60 \mathrm{~km}$, so this effect is believed to be statistically significant. It is therefore recommended that models with a horizontal gridbox size greater than $60 \mathrm{~km}$ use a value of $d=60 \mathrm{~km}$ in (11). The dashed lines in Fig. 7a become horizontal where this occurs.

The $\Delta z_{0}$ data are reasonably well fitted by the following expression, shown by the dashed lines in Fig. 7b:

$$
\log _{10} \Delta z_{0}=0.3 \log _{10} d-0.031 s-0.315
$$


where $\Delta z_{0}$ is in $\mathrm{km}$. This expression is valid for all values of $d$ considered. It is interesting that for a horizontal gridbox size of less than $20 \mathrm{~km}$ the mean decorrelation length is always less than $1 \mathrm{~km}$ and in many cases is less than the likely model vertical resolution in the middle and upper troposphere. Given that most ice clouds would be more than two layers thick, it may be a reasonable approximation for some high resolution models to simply assume that ice cloud inhomogeneities are randomly overlapped (i.e. $\Delta z_{0}=0$ ).

We next consider the fractional variance and decorrelation length of the visible extinction coefficient, calculated from $Z$ using (9). As explained in section 3, $f_{\alpha}$ is simply 'scaled down' from $f_{\text {IWC }}$ according to the square of the ratio of the exponents in (9) and (8), yielding the following expression:

$$
\log _{10} f_{\alpha}=0.3 \log _{10} d-0.04 s-0.96
$$

The decorrelation lengths are found to be exactly the same as for IWC.

In section $4 \mathrm{~b}$ and Fig. $3 \mathrm{~d}$, an apparent dependence of $f_{\text {IWC }}$ on distance from cloud top and base was found. Although the minimum in $f_{\mathrm{IWC}}$ coincided approximately with the maximum in shear, as would be predicted by (11), we would expect a constant shear to still result in a decrease of $f_{\text {IWC }}$ with distance from cloud top, since the ice in the fallstreaks would get gradually more homogenized as it fell through the sheared region. To investigate this further we consider only cases of shear between 0 and $10 \mathrm{~m} \mathrm{~s}^{-1}$, and categorize each $f_{\text {IWC }}$ observation according to its distance above cloud base $z_{\mathrm{b}}$ and its distance below cloud top $z_{\mathrm{t}}$. Cloud boundaries are deemed to be where the cloud fraction falls below unity. The solid lines in Fig. 8 depict mean $f_{\text {IWC }}$ versus $z_{\mathrm{b}}$ for clouds of thickness ranging from 0.3 to $3.6 \mathrm{~km}$. Thicker clouds have a much noisier profile due to their less frequent occurrence in this dataset, so have not been plotted. Panels a and b correspond to gridbox size ranges of 5-10 km and 40-80 km, respectively.

A clear tendency is evident for the fractional variance to decrease steadily from cloud top to perhaps half of its cloud-top value around two-thirds of the depth into the cloud, and then increase toward cloud base. Generally the fractional variance at cloud top is greater than that at cloud base. The minimum fractional variance of clouds $3.6 \mathrm{~km}$ thick is found to be around a third that 
of clouds $0.3 \mathrm{~km}$ thick. The $d^{0.3}$ dependence of $f_{\text {IWC }}$ in (11) implies a factor of 1.87 difference in $f_{\text {IWC }}$ between the data plotted in Figs. $8 \mathrm{a}$ and $8 \mathrm{~b}$, which is consistent with the observations. Nonetheless, the scatter for $40-80 \mathrm{~km}$ gridboxes is considerably more than that for $5-10 \mathrm{~km}$ gridboxes.

The dashed lines in Fig. 8 show the following fit to the data:

$$
f_{\text {IWC }}=0.153 \bar{d}^{0.03} R\left(z_{\mathrm{b}}, z_{\mathrm{t}}\right)
$$

where

$$
\begin{aligned}
R & =\exp \left[-1.5 z_{\mathrm{b}}-0.2 z_{\mathrm{t}}\right] \\
& +\exp \left[-1.5 z_{\mathrm{t}} /\left(z_{\mathrm{b}}+z_{\mathrm{t}}\right)-0.05 z_{\mathrm{b}}\right]
\end{aligned}
$$

The terms on the right hand side of (15) may be explained as follows. The first term expresses the inverse-exponential dependence of $f_{\text {IWC }}$ on distance from cloud base, with the $-0.2 z_{\mathrm{t}}$ term in the exponent describing how the cloud-base value of $f_{\text {IWC }}$ decreases with increasing cloud thickness. The second exponential expresses the inverse-exponential dependence of $f_{\text {IWC }}$ on the fraction of the distance into the cloud from cloud top, with the $-0.05 z_{\mathrm{b}}$ term describing the decrease in the cloud top value with increasing cloud thickness.

The full expression including dependence on gridbox size, wind shear and distance from the cloud boundaries is then

$$
\log _{10} f_{\mathrm{IWC}}=0.3 \log _{10} d+\log _{10} R-0.04 s-0.66
$$

Similarly for the fractional variance of visible extinction coefficient we have:

$$
\log _{10} f_{\alpha}=0.3 \log _{10} d+\log _{10} R-0.04 s-0.69 \text {. }
$$

As with (11) and (13), a maximum value of $d=60 \mathrm{~km}$ should be used in (16) and (17). No dependence of $\Delta z_{0}$ on the position in the cloud was found.

The fractional variance data were examined as a function of both temperature and absolute altitude, but no significant dependence was found. It should be noted that when the weak temperature 
dependence of the $b$ coefficient in (7) is included in the retrieval of IWC from $Z$, then this can feed through directly to a weak temperature dependence of $f_{\text {IWC. }}$. However, since the temperature dependence of $b$ is due to non-Rayleigh scattering of the larger particles at $94 \mathrm{GHz}$, rather than a meteorological phenomenon, and the fact that there are inherent uncertainties in our representation of non-Rayleigh effects, we are not confident that this represents a genuine temperature dependence of $f_{\text {IWC }}$. This finding is not at odds with the previous finding of a strong temperature dependence of absolute IWC (e.g. Stephens et al. 1990; McFarquhar and Heymsfield 1997), and certainly there is a robust temperature dependence of $c$ in (7) for all radar frequencies (Liu and Illingworth 2000), but as discussed in section $3 \mathrm{~b}$, this does not affect the retrieval of $f_{\text {IWC }}$.

\section{Conclusions}

A large dataset of ice cloud observations by cloud radar has been used to derive essential information on cloud variability for use in the next generation of cloud schemes in operational numerical models. It is found that observed IWC distributions are usually well represented by lognormal or gamma distributions. Expressions have been fitted relating the fractional variances of IWC and $\alpha$ to horizontal gridbox size, wind shear and distance above cloud base and below cloud top.

A clear tendency for increased shear to cause a homogenization of the cloud was evident, which resulted in significantly lower fractional variance. Due to the vertical distance over which the homogenization occurs, fractional variance tended to be greatest at cloud top and decrease with depth into the cloud, although beginning to increase again in the lowest third of the cloud.

In low shear conditions the vertical decorrelation length, which is independent of the parameter being correlated, was found to vary between $0.5 \mathrm{~km}$ for a $2 \mathrm{~km}$ gridbox to $2 \mathrm{~km}$ for a $300 \mathrm{~km}$ gridbox. Again, shear was found to reduce the correlation, principally because of the change in fallstreak orientation. Both fractional variance and vertical decorrelation length increased as gridbox size to the power of 0.3 .

Some radiation schemes under development are able to represent the sub-gridscale variability of cloud properties, so would be able to make use of the results on fractional variance and vertical 
decorrelation reported in this paper. They typically split each column of gridboxes up into a number of narrower columns and perform radiative transfer calculations separately for each one. This way an arbitrary sub-gridscale distribution of cloud properties may be specified, and partially cloudy gridboxes treated in a more consistent way. To run the full radiation scheme on each subcolumn would be very computationally expensive, but by simply running each different wave-band of the radiation scheme over a different sub-column, no significant increase in cost is incurred $(\mathrm{H}$. W. Barker, personal communication).

It is not immediately clear how these results should be applied in partially cloudy models gridboxes. We excluded partially cloudy gridboxes from the analysis because to include them would imply that the cloud-free regions were distributed evenly through the gridbox, in the same way as in fields of cumulus or broken stratocumulus. In the case of ice clouds, partially cloudy gridboxes are invariably those containing the edges of a large cloud that may span many gridboxes in the horizontal. Therefore the best approach for a model may be to retain a parameterization for cloud fraction, and apply the formula for fractional variance to only the cloudy part of the box, using a value of $d$ that reflects the size of the cloudy sector of the box, rather than the size of the gridbox as a whole.

The large majority of the clouds considered in this study would have been nucleated from cloud top in small generating cells, so it would be useful to extend this study to the tropics where a most ice clouds are anvil cirrus. It should also be pointed out that the relationships derived here apply principally to stratiform clouds, and model gridboxes containing storm cells penetrating into more extensive ice cloud should treat the overlap of the convective and stratiform regions separately, with the convective component presumably conforming to maximum overlap. The forthcoming NASA CloudSat mission involves launching a 94-GHz radar in space in 2004 and will be able to provide data on IWC variability and overlap on a global scale.

Acknowledgements. We are grateful to the Radio Communications Research Unit at the Rutherford Appleton Laboratory, UK, for providing the Galileo radar and drop-counting rain-gauge data. The Galileo radar was developed for the European Space Agency by Officine Galileo, the Ruther- 
ford Appleton Laboratory and the University of Reading. The EUCREX data were provided by Phil Brown and the Met Office mesoscale model data over Chilbolton were provided by Peter Clark. This research received funding from the European Union CloudNET project (grant EVK2CT-2000-00065) and NERC grant NER/T/S/01023.

\section{REFERENCES}

Barker, H. W., B. A. Wielicki, L. Parker, 1996: A parameterization for computing grid-averaged solar fluxes for inhomogeneous marine boundary-layer clouds. 2: Validation using satellite data. J. Atmos. Sci., 53(16), 2304-2316.

Brown, P. R. A., and P. N. Francis, 1995: Improved measurements of the ice water content in cirrus using a total-water probe. J. Atmos. Oceanic Technol., 12(2), 410-414.

Brown, P. R. A., A. J. Illingworth, A. J. Heymsfield, G. M. McFarquhar, K. A. Browning and M. Gosset, 1995: The role of spaceborne millimeter-wave radar in the global monitoring of ice-cloud. J. Appl. Meteorol., 34(11), 2346-2366.

Cahalan, R. F., W. Ridgeway, W. J. Wiscombe, T. L. Bell and J. B. Snider, 1994: The albedo of fractal stratocumulus clouds. J. Atmos. Sci., 51, 2434-2455.

Donner, L. J., C. J. Seman, B. J. Soden, R. S. Hemler, J. C. Warren, J. Strom and K.-N. Liou, 1997: Large-scale ice clouds in the GFDL SKYHI general circulation model. J. Geophys. Res., 102, $21745-21768$.

Fox, N. I., and A. J. Illingworth, 1997: The potential of a spaceborne radar for the detection of stratocumulus clouds. J. Appl. Meteorol., 36(6), 676-687.

Francis, P. N., P. Hignett and A. Macke, 1998: The retrieval of cirrus cloud properties from aircraft multi-spectral reflectance measurements during EUCREX'93. Quart. J. Roy. Meteorol. Soc., 124, 1273-1291.

Fu, Q., B. Carlin and G. Mace, 2000: Cirrus horizontal inhomogeneity and OLR bias. Geophys. Res. Lett., 27(20), 3341-3344. 
Heymsfield, A. J., and D. G. Baumgardner, 1985: Summary of workshop on processing 2-D probe data. Bull. Am. Meteorol. Soc., 66, 437-440.

Hogan, R. J., and A. J. Illingworth, 1999: The potential of spaceborne dual-wavelength radar to make global measurements of cirrus clouds. J. Atmos. Oceanic Technol., 16(5), 518-531.

Hogan, R. J., and A. J. Illingworth, 2000: Deriving cloud overlap statistics from radar. Quart. J. Roy. Meteorol. Soc., 126(569A), 2903-2909.

Hogan, R. J., C. Jakob and A. J. Illingworth, 2001: Comparison of ECMWF cloud fraction with radar-derived values. J. Appl. Meteorol., 40(3), 513-525.

Larson, V. E., R. Wood, P. R. Field, J.-C. Golaz, T. H. Vonder Haar and W. R. Cotton, 2001: Systematic biases in the microphysics and thermodynamics of numerical models that ignore subgrid-scale variability. J. Atmos. Sci., 58, 1117-1128.

Liang, X.-Z., and W. C. Wang, 1997: Cloud overlap effects on general circulation model simulations. J. Geophys. Res., 102, 11 039-11047.

Liou, K.-N., 1986: Influence of cirrus clouds on weather and climate processes: A global perspective. Monthly Weather Rev., 114, 1167-1199.

Liou, K.-N., and N. Rao, 1996: Radiative transfer in cirrus clouds. 4: On cloud geometry, inhomogeneity and absorption. J. Atmos. Sci., 53, 3046-3065.

Liu, C.-L. and A. J. Illingworth, 2000: Towards more accurate retrievals of ice water content from radar measurement of clouds. J. Appl. Meteorol., 39, 1130-1146.

Lohmann, U., and Roeckner, E., 1996: Design and performance of a new cloud microphysics scheme developed for the ECHAM general circulation model. Clim. Dyn., 12, 557572.

Mace, G. G., C. Jakob and K. P. Moran, 1998: Validation of hydrometeor occurrence predicted by the ECMWF using millimeter wave radar data. Geophys. Res. Lett., 25(10), 1645-1648.

McFarquhar, G. M., and A. J. Heymsfield, 1997: Parameterization of tropical cirrus ice crystal 
size distributions and implications for radiative transfer: Results from CEPEX. J. Atmos. Sci., 54(18), 2187-2200.

Morcrette, J. J., and Y. Fouquart, 1986: The overlapping of cloud layers in shortwave radiation parameterization. J. Atmos. Sci., 43, 321-328.

Pomroy, H. R., and A. J. Illingworth, 2000: Ice cloud inhomogeneity: Quantifying bias in emissivity from radar observations. Geophys. Res. Lett., 27(14), 2101-2104.

Slingo, J. M., 1987: The development and verification of a cloud prediction scheme for the ECMWF model. Quart. J. Roy. Meteorol. Soc., 115, 899-927.

Smith, R. N. B., 1990: A scheme for predicting layer clouds and their water content in a general circulation model. Quart. J. Roy. Meteorol. Soc., 116, 435-460.

Smith, S. A., and A. D. DelGenio, 2001: Analysis of aircraft, radiosonde, and radar observations in cirrus clouds observed during FIRE II: The interactions between environmental structure, turbulence, and cloud microphysical properties. J. Atmos. Sci., 58, 451-461.

Stephens, G. L., S. C. Tsay, P. W. Stackhouse Jr. and P. J. Flatau, 1990: The relevance of the microphysical and radiative properties of cirrus clouds to climate and climatic feedback. $J$. Atmos. Sci., 47, 1742-1752.

Tiedtke, M., 1993: Representation of clouds in large-scale models. Quart. J. Roy. Meteorol. Soc., 104, 677-690.

Tiedtke, M., 1996: An extension of cloud-radiation parameterization in the ECMWF model: The representation of subgrid-scale variations of optical depth. Mon. Weath. Rev., 124(4), 745750.

Tompkins, A. M., 2002: A prognostic parameterization for the subgrid-scale variability of water vapor and clouds in large-scale models and its use to diagnose cloud cover. J. Atmos. Sci., 59(12), 1917-1942. 


\section{List of Figures}

1 Retrieval of $Z$-IWC power law from EUCREX data. The light gray points in panel a indicate the individual 5-s samples, and the thin solid lines indicate the SD lines for each of the 115 horizontal aircraft runs in EUCREX with their length indicating \pm 1 standard deviation. Panel $\mathrm{b}$ depicts a histogram of the slopes of these lines, and the 'best' SD line, with a slope equal to the mean of the slopes of the individual runs, is shown by the thick solid line in panel a. The thick dashed line shows the regression, and indicates the difference in slope that can occur when the parameters are not perfectly correlated. Note that $Z$ is plotted in logarithmic $\mathrm{dBZ}$ units, where $Z_{\mathrm{dBZ}}=10 \log _{10}\left(Z_{\mathrm{mm}^{6} \mathrm{~m}^{-3}}\right) \ldots \ldots \ldots 26$

2 As Fig. 1, but for visible extinction coefficient $\alpha \ldots \ldots \ldots$

3 (a) Radar reflectivity factor measured by the Galileo radar on 27 December 1999, and (b) IWC calculated from it in 0.3 -km layers using the $Z$-IWC relationship in (8). The dashed line at an altitude of $6.9 \mathrm{~km}$ in panel b shows the division between the 'low shear' and 'high shear' cases considered in the text and in Fig. 6. Panel c shows the zonal $(u)$ and meridional $(v)$ wind components at 11 UTC over Chilbolton according to the mesoscale version of the UK Met Office Unified Model. Panel d depicts the mean fractional variance of IWC at each height, calculated from 50-km horizontal samples of cloud, and using the wind speed from panel $\mathrm{c}$ to determine the appropriate sample time at each height. . . . . . . . 
Probability distribution functions of ice water content in three boxes measuring $50 \mathrm{~km}$ in the horizontal and $0.3 \mathrm{~km}$ in the vertical, taken from the data shown in Fig. 3 at altitudes of $4.65,6.45$ and $8.25 \mathrm{~km}$. The dwell times required to sample the equivalent of $50 \mathrm{~km}$ were determined from the wind speed over Chilbolton as reported by the mesoscale version of the Met Office model, and each box was centered on 1230 UTC. Overlayed on each panel are lognormal and gamma distributions fitted such that they have the same mean and variance as the data. The $f_{\text {IWC }}$ parameter (defined in Eq. 1). is also indicated. . . . . . . . . . . 28

5 As Fig. 4, but for boxes measuring $250 \mathrm{~km}$ in the horizontal. . . . . . . . . . 28

6 The circles depict the mean correlation coefficient of the ice water content in $50 \times 0.3-\mathrm{km}$ boxes, versus vertical separation. Panel a shows the results for the data between 6.9 and $8.9 \mathrm{~km}$ in Fig. 3 (low shear case), and panel b shows the results for the data between 4.9 and $6.9 \mathrm{~km}$ (high shear case). The error bars indicate the standard deviation of the raw data. The solid and dashed lines show exponential and linear fits to the first point at $\Delta z=0.3 \mathrm{~km}$, as discussed in the text. 29

7 The parameters $f_{\mathrm{IWC}}$ and $\Delta z_{0}$ (averaged from 18 months of cloud radar data) versus horizontal gridbox size and the magnitude of the vertical wind shear $s$. The dashed lines show the analytical fits given in (11) and (12). The error bars indicate the range of raw values from which the means were calculated, for shear in the range $5-10 \mathrm{~m} \mathrm{~s}^{-1} \mathrm{~km}^{-1}$. The spread is similar for other values of shear. . . . . .

8 The parameter $f_{\text {IWC }}$ versus distance from cloud base $z_{\mathrm{b}}$, for two different gridbox size ranges (panels a and b). Each line corresponds to clouds of a different depth, the solid lines being the means of the retrieved values, and the dashed lines being $0.28 R$ in panel a and $0.52 R$ in panel $\mathrm{b}$, where $R$ is given by (15) . . . . . . 31 

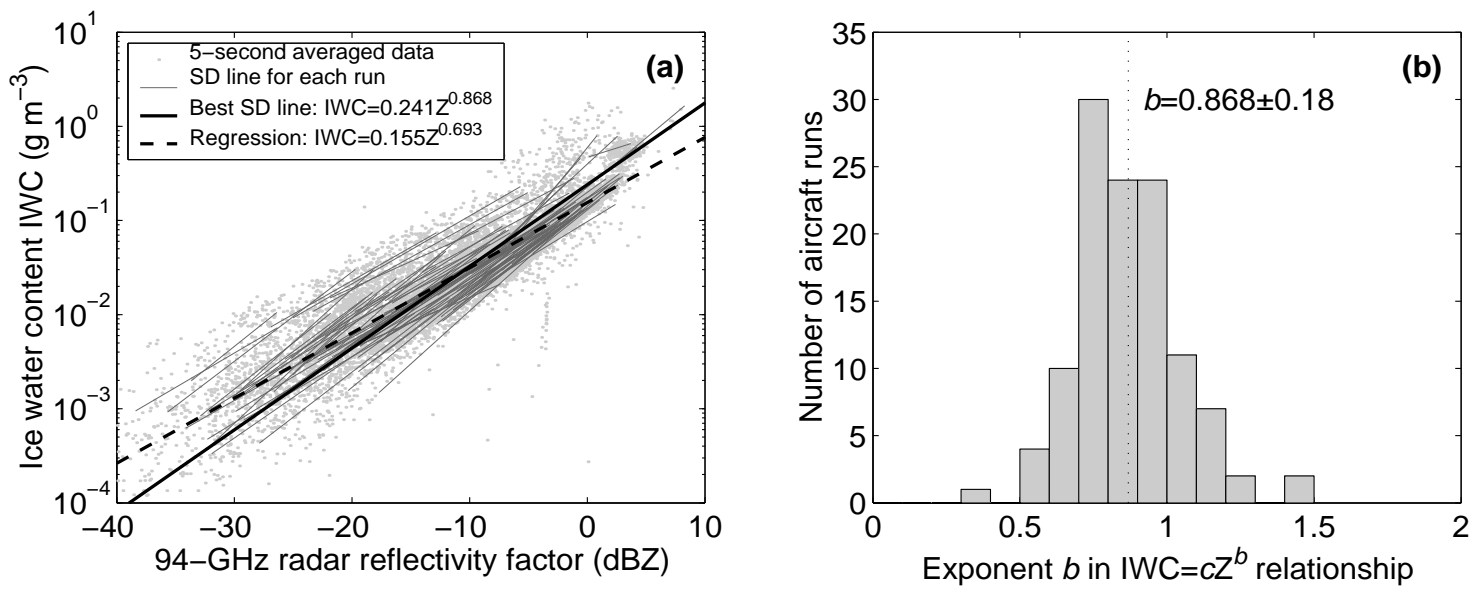

FIG. 1: Retrieval of Z-IWC power law from EUCREX data. The light gray points in panel a indicate the individual 5-s samples, and the thin solid lines indicate the SD lines for each of the 115 horizontal aircraft runs in EUCREX with their length indicating \pm 1 standard deviation. Panel b depicts a histogram of the slopes of these lines, and the 'best' SD line, with a slope equal to the mean of the slopes of the individual runs, is shown by the thick solid line in panel a. The thick dashed line shows the regression, and indicates the difference in slope that can occur when the parameters are not perfectly correlated. Note that $Z$ is plotted in logarithmic dBZ units, where $Z_{\mathrm{dBZ}}=10 \log _{10}\left(Z_{\mathrm{mm}^{6} \mathrm{~m}^{-3}}\right)$.
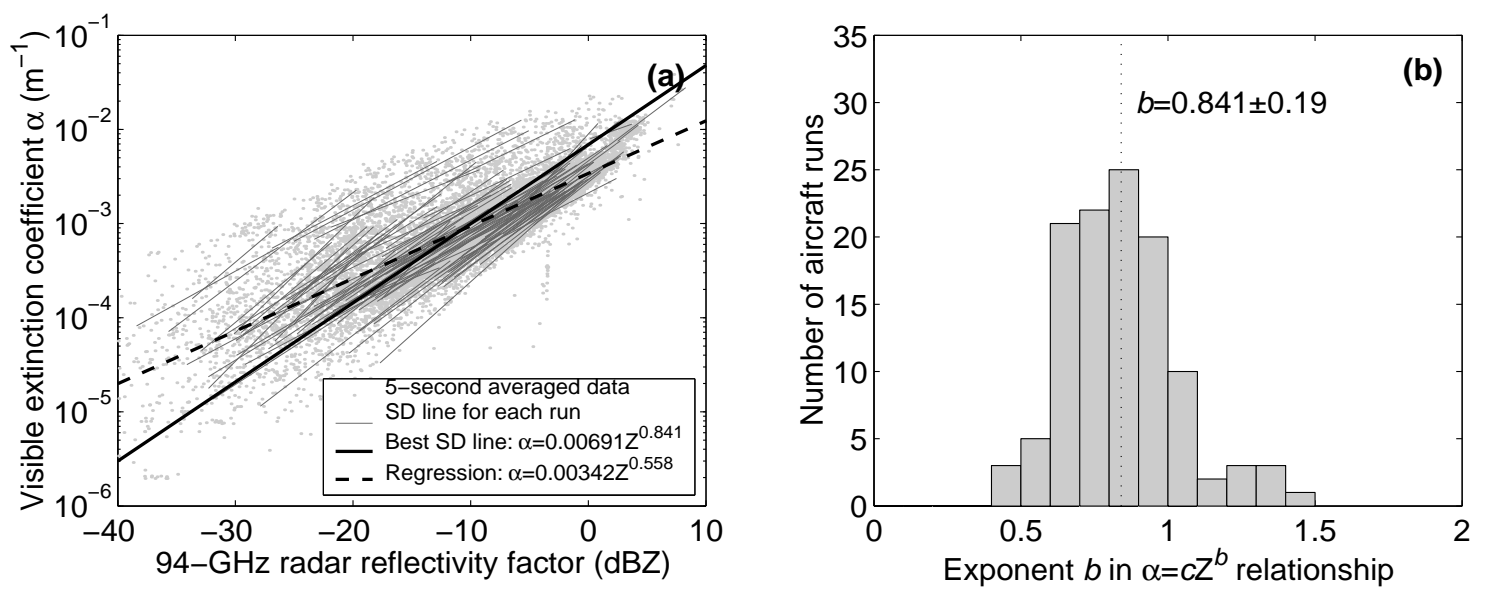

FIG. 2: As Fig. 1, but for visible extinction coefficient $\alpha$. 

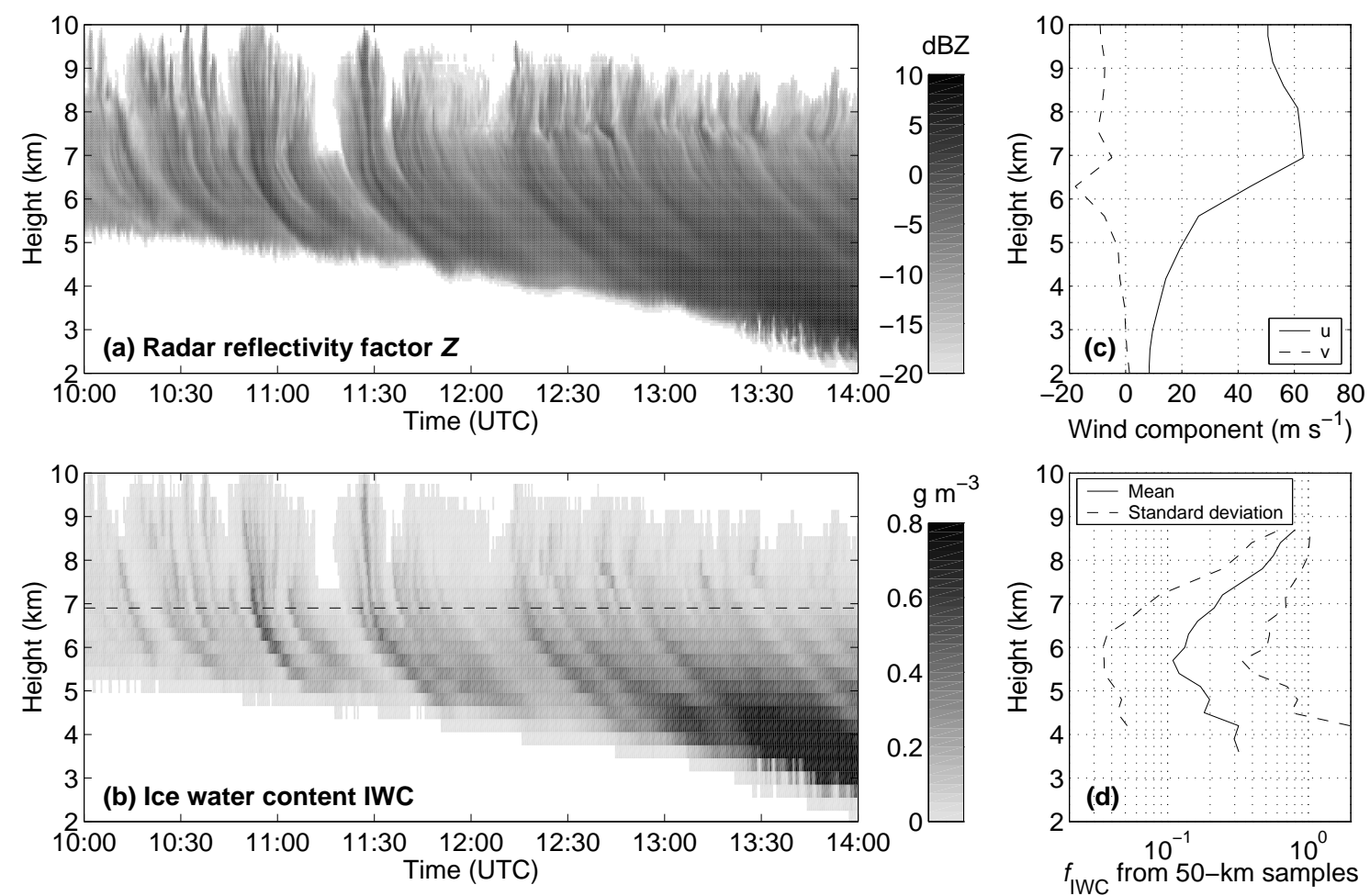

FIG. 3: (a) Radar reflectivity factor measured by the Galileo radar on 27 December 1999, and (b) IWC calculated from it in 0.3-km layers using the $Z$-IWC relationship in (8). The dashed line at an altitude of $6.9 \mathrm{~km}$ in panel $\mathrm{b}$ shows the division between the 'low shear' and 'high shear' cases considered in the text and in Fig. 6. Panel $\mathrm{c}$ shows the zonal $(u)$ and meridional $(v)$ wind components at 11 UTC over Chilbolton according to the mesoscale version of the UK Met Office Unified Model. Panel d depicts the mean fractional variance of IWC at each height, calculated from 50-km horizontal samples of cloud, and using the wind speed from panel c to determine the appropriate sample time at each height. 

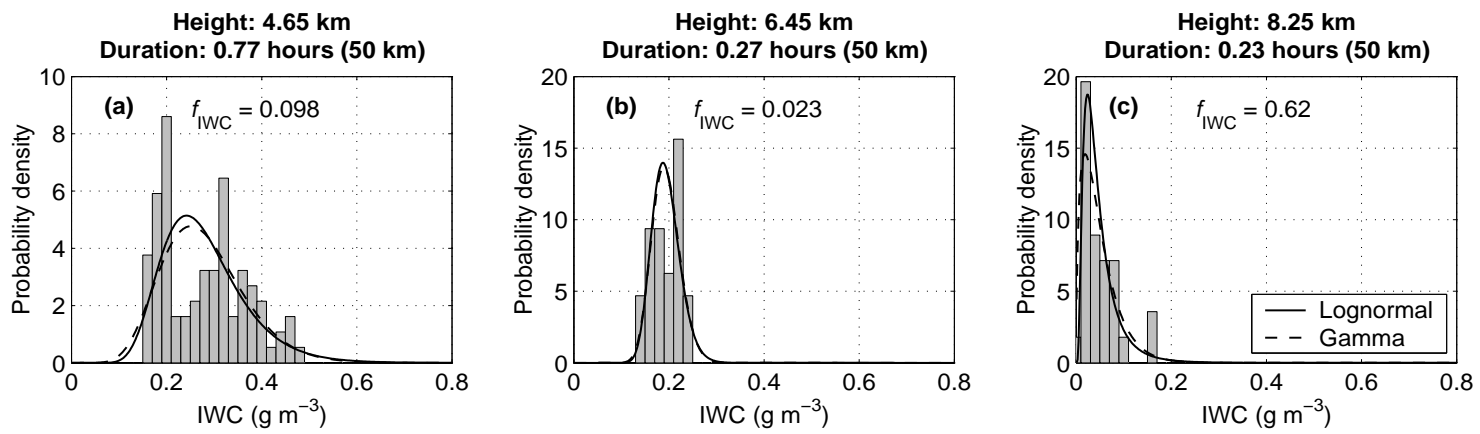

FIG. 4: Probability distribution functions of ice water content in three boxes measuring $50 \mathrm{~km}$ in the horizontal and $0.3 \mathrm{~km}$ in the vertical, taken from the data shown in Fig. 3 at altitudes of 4.65, 6.45 and $8.25 \mathrm{~km}$. The dwell times required to sample the equivalent of $50 \mathrm{~km}$ were determined from the wind speed over Chilbolton as reported by the mesoscale version of the Met Office model, and each box was centered on 1230 UTC. Overlayed on each panel are lognormal and gamma distributions fitted such that they have the same mean and variance as the data. The $f_{\text {IWC }}$ parameter (defined in Eq. 1). is also indicated.
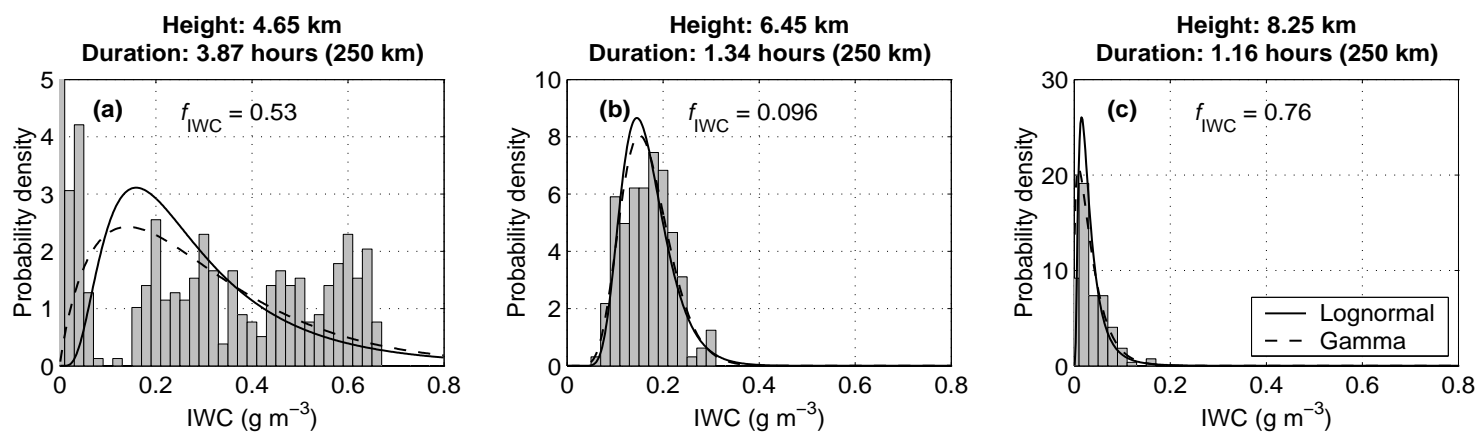

FIG. 5: As Fig. 4, but for boxes measuring $250 \mathrm{~km}$ in the horizontal. 

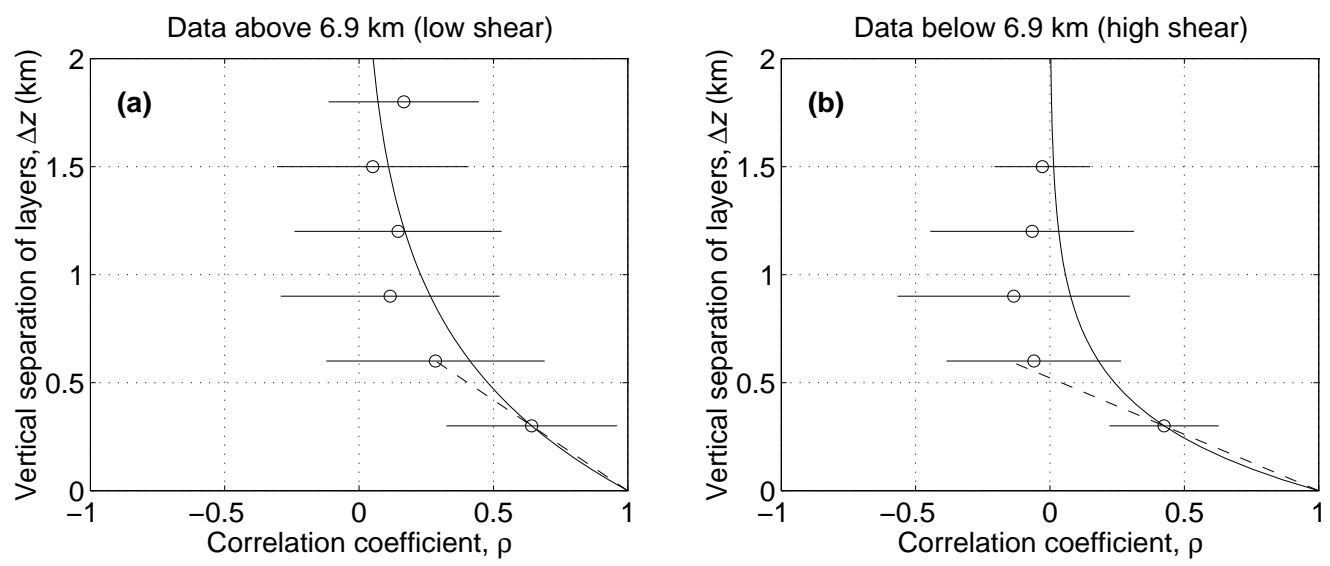

FIG. 6: The circles depict the mean correlation coefficient of the ice water content in $50 \times 0.3-\mathrm{km}$ boxes, versus vertical separation. Panel a shows the results for the data between 6.9 and $8.9 \mathrm{~km}$ in Fig. 3 (low shear case), and panel b shows the results for the data between 4.9 and $6.9 \mathrm{~km}$ (high shear case). The error bars indicate the standard deviation of the raw data. The solid and dashed lines show exponential and linear fits to the first point at $\Delta z=0.3 \mathrm{~km}$, as discussed in the text. 

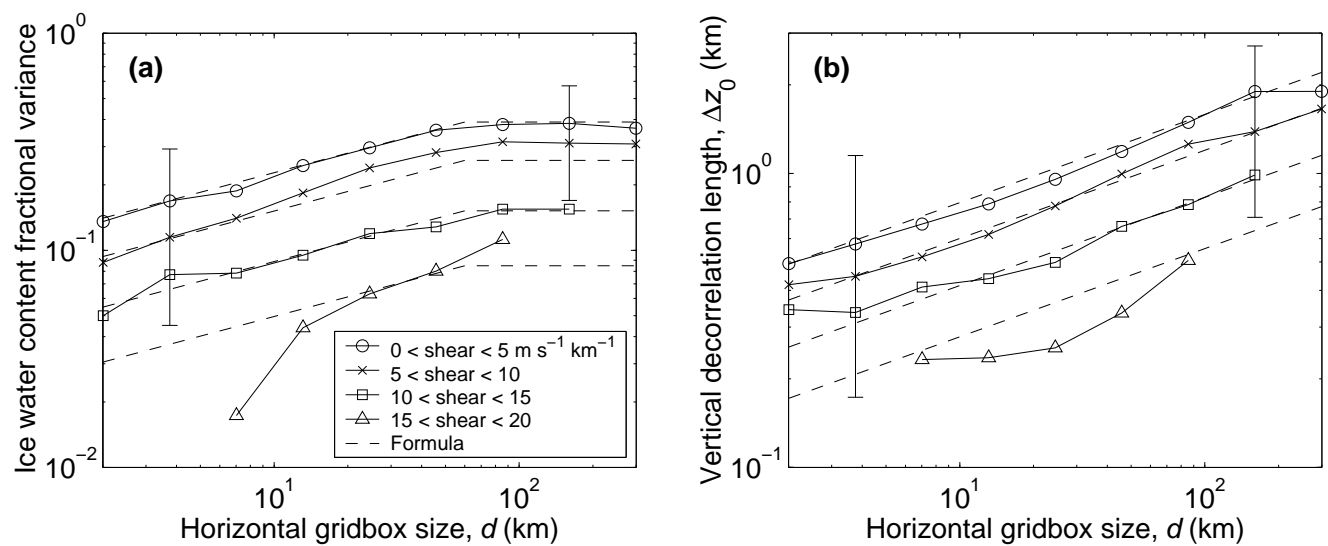

FIG. 7: The parameters $f_{\text {IWC }}$ and $\Delta z_{0}$ (averaged from 18 months of cloud radar data) versus horizontal gridbox size and the magnitude of the vertical wind shear $s$. The dashed lines show the analytical fits given in (11) and (12). The error bars indicate the range of raw values from which the means were calculated, for shear in the range $5-10 \mathrm{~m} \mathrm{~s}^{-1} \mathrm{~km}^{-1}$. The spread is similar for other values of shear. 

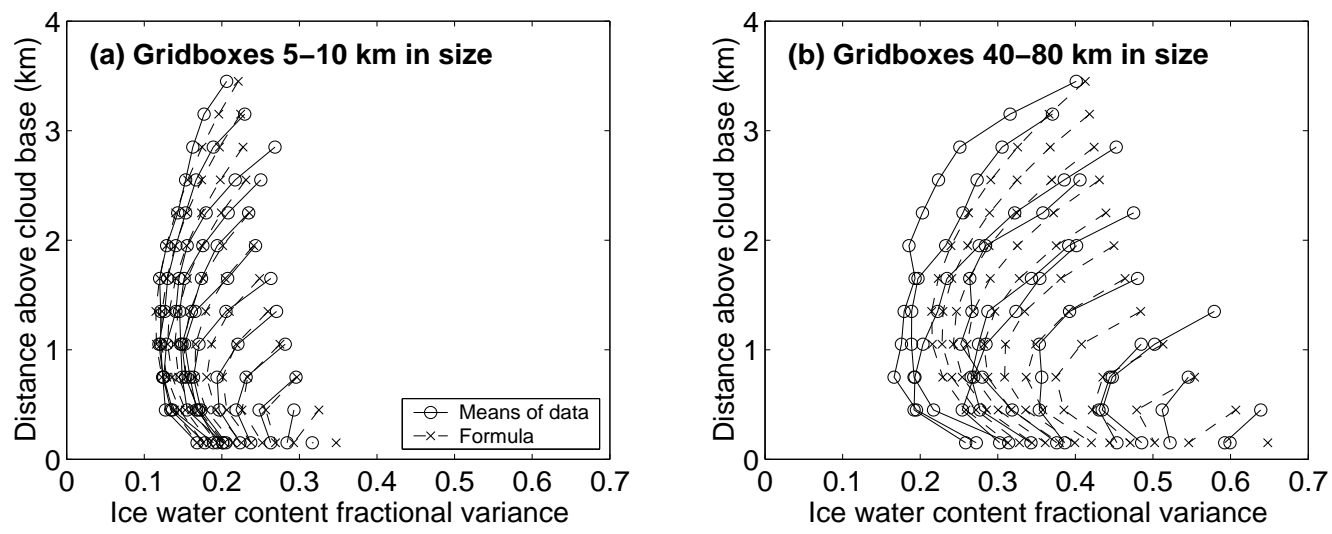

FIG. 8: The parameter $f_{\text {IWC }}$ versus distance from cloud base $z_{\mathrm{b}}$, for two different gridbox size ranges (panels a and b). Each line corresponds to clouds of a different depth, the solid lines being the means of the retrieved values, and the dashed lines being $0.28 R$ in panel a and $0.52 R$ in panel $\mathrm{b}$, where $R$ is given by (15). 